\title{
Diacronie
}

Studi di Storia Contemporanea

$N^{\circ} 35,3 \mid 2018$

Gli strumenti di Clio

\section{Commento di Paola Bernasconi a Un'età contro la storia. Saggio sulla rivoluzione del XXI secolo}

\section{Paola Bernasconi}

\section{(2) OpenEdition}

\section{Journals}

\section{Edizione digitale}

URL: http://journals.openedition.org/diacronie/9069

DOI: 10.4000/diacronie.9069

ISSN: 2038-0925

\section{Editore}

Association culturelle Diacronie

\section{Notizia bibliografica digitale}

Paola Bernasconi, «Commento di Paola Bernasconi a Un'età contro la storia. Saggio sulla rivoluzione del XXI secolo », Diacronie [Online], № 35, 3 | 2018, documento 9, Messo online il 29 septembre 2018, consultato il 02 mai 2019. URL : http://journals.openedition.org/diacronie/9069; DOI : 10.4000/ diacronie.9069 


\section{Diacronie}

Studi di Storia Contemporanea

\section{$35,3 / 2018$}

Gli strumenti di Clio: uomini, luoghi e teorie della storia dalla tradizione critica alla comunicazione digitale

\section{Commento di Paola Bernasconi a Un'età contro la storia. Saggio sulla rivoluzione del XXI secolo}

\section{Paola BERNASCONI}

Per citare questo articolo:

BERNASCONI, Paola, «Commento di Paola Bernasconi a Un'età contro la storia. Saggio sulla rivoluzione del XXI secolo», Diacronie. Studi di Storia Contemporanea : Gli strumenti di Clio: uomini, luoghi e teorie della storia dalla tradizione critica alla comunicazione digitale, 35, 3/2018, 29/09/2018,

URL: < http://www.studistorici.com/2018/09/29/bernasconi_numero_35/ >

Diacronie Studi di Storia Contemporanea $\rightarrow$ http://www.diacronie.it Rivista storica online. Uscita trimestrale.

redazione.diacronie@hotmail.it

Comitato di direzione: Naor Ben-Yehoyada - João Fábio Bertonha - Christopher Denis-Delacour - Maximiliano Fuentes Codera Anders Granås Kjøstvedt - John Paul Newman - Deborah Paci - Niccolò Pianciola - Spyridon Ploumidis - Wilko Graf Von Hardenberg

Comitato di redazione: Jacopo Bassi - Luca Bufarale - Gianluca Canè - Fausto Pietrancosta - Alessandro Salvador - Matteo Tomasoni Diritti: gli articoli di Diacronie. Studi di Storia Contemporanea sono pubblicati sotto licenza Creative Commons 3.0. Possono essere riprodotti e modificati a patto di indicare eventuali modifiche dei contenuti, di riconoscere la paternità dell'opera e di condividerla allo stesso modo. La citazione di estratti è comunque sempre BY SA autorizzata, nei limiti previsti dalla legge. 


\title{
9/ Commento di Paola Bernasconi a Un'età contro la storia. Saggio sulla rivoluzione del XXI secolo
}

\author{
Paola BERNASCONI
}

"Dove collocare lo spazio per la memoria in un siffatto sistema di comunicazione? Quale memoria?" Paola

Bernasconi nel suo commento al saggio di Giuseppe Carlo Marino su globalizzazione e crisi del "pensare storico" prova a rispondere a queste domande interrogandosi sui mutamenti che la rivoluzione elettronico-informatica e le dinamiche poltico-economiche hanno impresso sul valore della cultura storica per la società globallizzata.

\section{Premessa}

Attendevo con grande curiosità la riflessione del Professor Marino sulla crisi della storia che sapevo essere una riflessione in fieri ma ben puntata su alcuni punti saldi. Punti che ho piacevolmente ritrovato nelle sue pagine pubblicate sotto forma di e-book, come compete ad un lavoro nei tempi della rivoluzione "elettronico-informatica".

Premetto che, lusingata dall'invito del Professor Marino a prendere parte a questa tavola rotonda virtuale, ho, tuttavia, accolto tale invito - ponendomi di fronte alla profonda riflessione sul senso della storia, prima ancora che sulla sua crisi - con lo stato d'animo di Zeno Contini di fronte alla sua ultima sigaretta. Come il personaggio Sveviano mi riprometto di smettere di occuparmi della storia, dicendo che sarà l'ultima volta ma, come lui fallisco di fronte alla mia inettitudine di conformarmi al "pensiero unico". Scelta questa via interpretativa il rischio è quello di affrontare l'argomento oggetto di questa riflessione in una chiave intimistica e polemica, di chi, scegliendo di occuparsi di storia, vive in un tempo che prende le distanze dal proprio passato, un po' come un operaio che si ritrovi senza fabbrica.

Di difficile collocazione, il saggio di Marino non sembra essere un saggio storico né storiografico, ma una riflessione di grande attualità e per certi versi premonitrice. Sicuramente stimolo per una riflessione sul nostro tempo. Due sono i concetti chiave che ho identificato nella mia riflessione: la crisi della storia e la rivoluzione in atto. 


\section{Crisi della storia}

La crisi della storia sembra essere un argomento di dibattito decisamente attuale, come dimostra il libro di Francesco Pitocco Crisi della storia, crisi della civiltà europea ${ }^{1}$, del 2012 e rimesso recentemente in commercio, sull'opera di Marc Bloch e quello, più recente di Giuseppe Galasso, Storia della storiografia italiana. Un profilo (Laterza 2017) in cui si identificano fattori di crisi in comune con la riflessione di Marino, dell'eccessivo tecnicismo e sul particolarismo della ricerca.

Qual è dunque il mestiere di storico? Marino definisce la nuova classe di storici come "semplici operai della storiografia" e produttori di quello che si può senza dubbio considerare "superfluo". Così come la classe operaia ha perso la sua connotazione nella storia, i nuovi storici si ritrovano a perseverare in un mestiere in crisi quanto il proprio oggetto di studio, come ben sottolinea il titolo di questo saggio. Non è, infatti, solo la storia a vivere il drammatico silenzio di questa crisi, che ben lontana sembra essere da qualsivoglia spirito rivoluzionario, bensì chi si è dedicato a tale mestiere.

Siamo altresì di fronte ad una produzione storiografica obbligata a piegarsi alle regole di mercato; per poter essere pubblicato, il lavoro degli storici deve essere in grado di "vendere" e quindi di avere un pubblico. Come un cane che si morde la coda, in un mercato in cui la ricerca del "benessere" individuale si basa su prodotti materiali ed il disinteresse verso la storia, e la cultura in genere, rappresenta un surplus secondario, tale mercato è naturalmente ristretto ed elitario non "necessitante" e l'operaio della storiografia si ritrova a produrre un bene non commerciabile, senza mercato.

\section{Rivoluzione elettronico-informatica ed il paradosso della globalizzazione}

Il grande paradosso della globalizzazione ha portato ad un acuirsi della percezione non solo della limitazione temporale, al presente, al momento, ma anche spaziale, al vicino, al locale, come vediamo riflesso nel rinnovato successo dei localismi, dei particolarismi, del campanilismo. Il luogo, come il tempo, viene percepito soprattutto nella sua vicinanza, nell'immediatezza. Denunciava Musil nell'“Uomo senza qualità", durante il manifetarsi degli effetti della precedente rivoluzione industriale, che: "abbiamo perso la capacità di osservare il mondo, sdraiati sotto un albero, attraverso le dita dei piedi", allo stesso modo, durante la rivoluzione in atto, stiamo

\footnotetext{
${ }^{1}$ PITOCCO, Francesco, Crisi della storia, crisi della civiltà europea, Milano-Roma, Mondadori-Università La Sapienza, 2012.

${ }^{2}$ GALASSO, Giuseppe, Storia della storiografia italiana. Un profilo, Bari-Roma, Laterza, 2017.
} 
perdendo la capacità di guardare il mondo da prospettive diverse, una di queste è la prospettiva storica, ma anche quella più ampia di apertuta generale verso le diversità che proprio la globalizzazione e le nuove forme di comunicazione pongono di fronte ai nostri occhi. Tale mi appare come un grande paradosso: «l'isolamento ed il frammento» che, come evidenzia Marino nel paragrafo sulla Fenomenologia della rivoluzione elettrico-informatica, citando il lavoro di Emanuele Severino ${ }^{3}$, «sono legati alla costrizione che con la propria potenza unisce i frammenti del mondo. Questo è il fondamento di ciò che viene chiamato 'globalizzazione'»"

L'ampiezza di informazioni che la rivoluzione tecnologico-informatica porta nelle nostre mani sembra creare un rigetto delle diversità che entrano nel nostro spazio attraverso il piccolo schermo da cui si osserva il mondo quasi a proteggere la solitudine individuale nell'interazione con l'altro e nell'immediatezza del presente. Quando il proprio vivere quotidiano si sente minacciato da una alterità che può mettere in discussione quel benessere lungamente agognato $\mathrm{e}$ falsamente raggiunto. Il successo dei localismi e l'insofferenza verso i migranti mostrano due realtà opposte contrapponendo il positivo della globalizzazione, l'apertura verso il mondo, ed i particolarismi della vita quotidiana.

Le «società destrutturate ed atomizzate»" ${ }^{5}$, eppure aperte all'omologazione, reagiscono alla vicinanza con il diverso respingendolo, per ritrovarsi in quella forma di identità che è il territorio riconoscibile, il proprio ambiente, come possiamo vedere dal successo di partiti politici regionalisti o dei movimenti indipendentisti. Il sistema fluido non coincide con la fluent society degli anni Sessanta. Furono proprio quegli anni a permettere alla generazione giovanile di aprirsi ad un mondo globale, attraverso uno scambio senza precedenti di informazioni su realtà lontane, permettendole di intervenire sulla storia con valori ideali condivisi. Si apriva, allora, una fase rivoluzionaria in seguito sconfitta dalla vittoria dell'ideologia occidentalista. Quei «soggetti collettivi», conferma Marino, «non sono benché minimamente comparabili con i cosiddetti movimenti» che prolificano da e sul web dando valore alle «soggettività individuali» come nel motto "uno vale uno".

Giunto al suo epilogo il sistema di divisioni fra classi, si può ipotizzare che la rivoluzione tecnologico-informatica produrrà un nuovo sistema di divisione sociale, non più basato sull'identificazione all'interno di un sistema produttivo industrializzato, bensì su una categorizzazione in base alle generazioni. La rottura fra generazioni propone una chiave interpretativa affascinante ${ }^{6}$ e può essere applicata in varie fasi del percorso storico: durante le lotte Risorgimentali così come nella crisi di fine secolo; durante il fascismo e nel dopoguerra ed in modo netto negli anni Sessanta. Tutti momenti "rivoluzionari" e di rottura. Nell'ottica di una

\footnotetext{
${ }^{3}$ SEVERINO, Emanuele, La potenza dell'errare. Sulla storia dell'Occidente, Milano, Rizzoli, 2014.

${ }^{4}$ MARINO, Giuseppe Carlo, Un'età contro la storia. Saggio sulla rivoluzione del XXI secolo, Palermo, Università degli Studi di Palermo, Dipartimento di Scienze Politiche e delle relazioni internazionali (DEMS), 2017, p. 35.

${ }^{5}$ Ibidem, p. 46

${ }^{6}$ MARINO, Giuseppe Carlo, Le generazioni italiane dall'Unità alla Repubblica, Milano, Bompiani, 2006.
} 
nuova fase rivoluzionaria ancora in fieri, che come evidenzia Marino, spezza quel filo di continuità con il passato, la distanza fra generazioni diventa abissale, perché prodotta da scoperte tecnologiche in rapidissima successione, tanto da lasciare indietro i vecchi schemi interpretativi della realtà circostante e delle forme di comunicazione. Le vecchie generazioni, per tenere il passo di fronte alle nuove tecnologie, dovranno imparare a codificare un linguaggio nuovo che le giovani generazioni già conoscono ma lo faranno comunque attraverso quel legame inscindibile con il loro tempo che diviene sempre più rapidamente "passato". Di contro, le nuove generazioni vanno perdendo l'interesse verso i vecchi linguaggi, le vecchie categorie interpretative della realtà e con esse, della storia. Una perdita inconsapevole di cui «le nuove generazioni ben difficilmente si avvedono, perché è impossibile accorgersi di un bene che non si è mai posseduto» ${ }^{7}$.

\section{Prospetto critico della rivoluzione del nostro tempo: un'improbabile fine} della storia

A partire dal 1989, l'universale "implosione di fine millennio" e la fine del comunismo hanno condotto alla morte di una dialettica che per più di un lungo secolo ha alimentato la riflessione sul destino dell'umanità («dialettica delle interferenze $\left.»^{8}\right)$. L'ideologia Occidentalista ha avuto il sopravvento aprendo la stagione della "libertà di consumare". Interessante è la considerazione data da Marino alla tesi del sociologo/politologo Francis Fukuyama che si è destreggiato in una postmoderna riflessione sulla fine della storia ${ }^{9}$, ipotizzando la sostituzione dell'essere umano con qualche prodotto della biotecnologia o della robotica. Un filone fantasy-politico (o cyberpunk) che tanto successo ha riscosso dal mercato dei sostenitori del superamento delle ideologie. Di fine delle ideologie si era già assoporato il fascino tra la fine degli anni Cinquanta e l'inizio degli anni Sessanta, con le prime intuizioni sulla società post-industriale, rivelandosi una tesi priva di fondamento come ha pienamente dimostrato il Sessantotto ed il decennio successivo dove le tensioni ideologiche hanno ritrovato un rinnovato momento ${ }^{10}$.

Un esperimento sulla teorizzazione degli usi/nuove possibilità offerte dalla supremazia tecnologico-informatica si può riscontrare nel pensiero di G. Casaleggio. Il fondatore "ideologico" del Movimento Cinque Stelle aveva teorizzato la supremazia della rete come mezzo per arrivare al controllo delle menti in funzione politica. Un documento di grande interesse è il video

\footnotetext{
${ }^{7}$ MARINO, Giuseppe Carlo, Un'età contro la storia. Saggio sulla rivoluzione del XXI secolo, cit., p. 53.

${ }^{8}$ Ibidem, p. 27

${ }^{9}$ FUKUYAMA, Francis, The end of history and the Last Man, New York, Free Press, 1992.

${ }^{10}$ BELL, Daniel, La fine dell'ideologia. Il declino delle idee politiche dagli anni Cinquanta ad oggi, Argomenti, Milano, SugarCo, 1991; la prima edizione americana è del 1960.
} 
sperimentale del 2008 del Progetto Gaia ${ }^{11}$ della Casaleggio Associati, sul potere della comunicazione. Partendo dal successo dell'impero romano che aveva creato una rete di comunicazione grazie alle strade, per arrivare all'uso della radio da parte di Mussolini il video delinea una storia in pillole buttata a caso sulla linea temporale.

Il vaneggiante messaggio che nella versione italiana parla con voce femminile di robot, recita: «Gaia, un nuovo ordine mondiale è nato oggi il 14 agosto 2054 ogni uomo è cittadino del mondo, soggetto alla stessa legge. Internet ha guidato il cambiamento diffondendo la comunicazione, il sapere e l'organizzazione a livello planetario, le piramidi di Egitto, il Colosseo di Roma, la Basilica della Santa Sapienza sono dimostrazioni di potere». Con la rete la comunicazione, la conoscenza e l'organizzazione non appartengono più al "potere" ma al popolo. Il video profetizza la creazione di un"'intelligenza collettiva delle persone in internet" (Brain Trust) che porterà alla distruzione dei simboli dell'Occidente ma anche alla sua affermazione tramite il trionfo della democrazia della rete. Nel 2040, dopo venti anni di distruzione dovuti ad una terza guerra mondiale e a varie catastrofi naturali resterà una selezionata popolazione mondiale di un miliardo di persone. A proposito dei localismi e della frantumazione della società «il nuovo ordine mondiale sarà guidato da movimenti dal basso per gestire i problemi locali», ma tali comunità saranno collegate dalla rete. L'esistenza umana sarà comprovata dall'iscrizione ad un social network mondiale che andrà a sostituire i documenti d'identità. Il completo compimento del progetto avverrà nel 2054, anno in cui cade il centenario dalla nascita di Casaleggio, in cui ci saranno le prime elezioni mondiali in rete e la creazione di un governo mondiale chiamato Gaia (la madre terra - l'Eden?) dove "ogni essere umano può diventare presidente" e controllare le azioni del governo tramite la rete. Si giungerà così alla fine delle ideologie. Il video finisce con un cervello luminoso che ruota.

Lo stesso Steve Jobs, il vero imprenditore del nuovo capitalismo tecnologico, ben aveva compreso l'andamento del nuovo mercato-natura. Il creare aspettativa verso l'ultimo modello dalla tecnologia sempre più perfezionata produceva (o produce) sul "libero consumatore" l'effetto di necessità improrogabile. Un effetto riscontrabile nelle lunghe file di fronte ai negozi che avrebbero commercializzato l'ultimo modello sul mercato. Un comportamento umano non dissimile dagli esempi riportati da Marino all'indomani della caduta del muro di Berlino, simbolo di una acquisita nuova libertà che seguiva e reagiva ad un periodo di lunghe privazioni definite "seduzioni di retroguardia".

La tecnologia ha creato un insieme di dipendenze difficilmente liquidabili come artificiali ma, appunto, "naturali", intese come status symbol di quella cornice in cui l'uomo tecnologico si ritrova prigioniero o libero; due condizioni che sembrano confondersi all'interno di questa "mutazione

\footnotetext{
${ }^{11}$ CASALEGGIO ASSOCIATI, Gaia - The future of politics, 21 ottobre 2008, URL:

<https://www.youtube.com/watch?v=sV8MwBXmewU> [consultato il 20 settembre 2018]. Qui si trova una versione italiana, URL: <https://www.youtube.com/watch?v=rx46BpHQ2mo> [consultato il 30 maggio 2018].
} 
antropologica" i cui effetti saranno oggetto di studio degli storici futuri (gli storici post moderni, o "post-umani" forse).

\section{Memoria: riflessioni su il tracollo della ragione dialettica e la crisi del "pensare storico" e della memoria}

La linearità della logica formale, in realtà, sembra spesso venire meno nei tempi della rivoluzione tecnologico informatica. Prendiamo come "luogo" della comunicazione infomatica ciò che avviene attraverso la rete, da distinguere dal "luogo fisico" della vita quotidiana, quello che come ho accennato precedentemente, corrisponde al territorio, al locale, al conosciuto.

Si è creato un dualismo spazio-temporale quasi bipolare. L'accelerazione del tempo tecnologico si riflette in una accelerazione del tempo umano creando una mancanza di momenti di scambio dialettico. Il linguaggio del web, e dei social come spazio comunicativo, deve seguire le regole della semplificazione, della velocità e dell'immediatezza: il discorso si sintetizza in frasi, spesso slogan, condivisi in un tempo $x$ in attesa di un'interazione in un tempo $y$. Spesso accade che $\mathrm{i}$ tempi di interazione tra parti del dialogo non siano sincronici per cui il tempo diventa fluido e personale anche in uno spazio esteso a livello mondiale quale è quello della rete. Ci sono studi, ad esempio, che si dedicano ad individuare il momento migliore per "postare" un articolo, prendendo l'esempio delle testate giornalistiche, in modo da raggiungere un numero maggiore di interlocutori. La rete ha le sue leggi. Spesso si punta sull'effetto, lo slogan, la grafica accattivante, l'immagine forte, la falsa notizia, per ottenere consensi quantificabili con i click che possono trasformarsi in profitto. Anche la rete ha le sue leggi di mercato.

Dove collocare lo spazio per la memoria in un siffatto sistema di comunicazione? Quale memoria?

Una memoria in pillole. Una memoria, strumentalizzata, tradita, in un sistema a-storico e spesso contro la storia. Una memoria in agonia, come la definisce Marino.

Se da una parte, la logica formale sopprime il contraddittorio, dall'altra ben altre contraddizioni rivela: quelle appunto, dei particolarismi in un'era globale e quelle che portano inevitabilmente alla degenerazione nel fanatismo ${ }^{12}$. La memoria è strettamente legata al "pensare storico" e nel prendere atto della sua agonia prendiamo atto della triste sorte della memoria. Avvertiva Bloch riguardo all'utilità della "memoria collettiva" per ogni società, che da una migliore conoscenza del passato otterrà una migliore capacità di risolvere i problemi del presente, ma anche di tali avvertimenti si è persa la memoria in chiave di una visione che punta al futuro, dimentica il proprio passato e schernisce il presente.

\footnotetext{
${ }^{12}$ MARINO, Giuseppe Carlo, Un'età contro la storia. Saggio sulla rivoluzione del XXI secolo, cit., p. 47.
} 
Marino identifica due ragioni principali per spiegare perché il "pensiero storico" non trova spazio nell'era della rivoluzione tecnologico informatica. Il primo è il tempo, inteso come tempo natura che ho interpretato come momento nella sua immediatezza. Il secondo è il declino della memoria. In questa corsa verso il "nuovo", ciò che riguarda il "vecchio" perde di importanza diventando "inutile e insignificante"così come il mito del futuro depriva il presente del suo significato lasciando l'uomo tecnologico senza «nessun radicamento profondo nel sapere e nella coscienza ${ }^{13}$. Di fronte ad una impossibile fine della storia, a vivere la vera crisi di inizio millennio è la memoria, una crisi pericolosa che come hanno evidenziato negli anni pensatori, storici e poeti, rischia di sciogliere quel collante che tiene insieme il nostro senso nel mondo.

\section{Conclusioni}

Ho dovuto leggere più volte il saggio del professor Marino per coglierne le sfumature e seguire il complesso ragionamento di un originale e coerente percorso intellettuale che viene sintetizzato in queste pagine. Ho cercato di interpretarne dei passaggi focalizzando la mia riflessione sulla rivoluzione tecnologico-informatica a discapito di altre interessanti interpretazioni come ad esempio quelle sul tempo e sul mercato. Il saggio richiede una lettura impegnativa perchè racchiude un'analisi complessa sul senso della storia e del percorso umano e, allo stesso tempo, apre molti interrogativi su un futuro che sembra segnato ma di cui è difficile ravvisarne i segni. Posso dire che conserverò con cura l'eredità di una fede combattuta e che all'ombroso Lucifero ${ }^{14}$ montaliano continuerò a contrapporre l'Angelo della storia con la speranza che osservi col suo stupore una minore quantità di macerie.

\footnotetext{
${ }^{13}$ Ibidem, p. 51.

${ }^{14}$ MONTALE, Eugenio, Piccolo Testamento, Milano, Atlas, 1953.
} 


\section{L'AUTORE}

Paola BERNASCONI è laureata in Storia Moderna e Contemporanea all'Università degli Studi di Roma "La Sapienza" con una tesi in storia dell'Europa dal titolo Da Auschwitz all'Europa. Simone Veil, primo presidente del Parlamento Europeo eletto a suffragio universale diretto; ha proseguito gli studi con un dottorato in Società, politica e istituzione in età contemporanea con una tesi sulla morte dello studente Paolo Rossi del 1966 dal titolo L'illusione democratica. Le associazioni studentesche prima del Sessantotto. Il caso di Roma. Ha collaborato con Il dizionario biografico degli italiani illustri dell'Enciclopedia G. Treccani per la stesura di biografie di esponenti del mondo politico e culturale dell'Italia dell'Ottocento e con l'Archivio storico della Camera dei Deputati su Il mito straniero nella costruzione dello Stato unitario. La sua area di specializzazione consiste nella storia del Novecento riguardo ai conflitti generazionali, $i$ processi di democratizzazione e la memoria collettiva. Ha preso parte al progetto sul Culto del Duce con Christopher Duggan e Stephen Gundle con una ricerca sul fondo archivistico "I sentimenti del Duce" da cui ha ricavato una ricerca sul mondo infantile durante il Regime: «A fairytale dictator: children's letters to the Duce», in Modern Italy, 18, 2/2013, pp. 129-140.

URL: < http://www.studistorici.com/progett/autori/\#Bernasconi > 